\title{
Incremento progresivo del índice de masa corporal en supervivientes pediátricos de linfoma
}

\section{Progressive increase in body mass index among short term survivors of pediatric lymphoma}

\author{
Mariana M. Rangel-Rivera ${ }^{1}$, Daphne MÉndez-Montoya ${ }^{2}$, Susana Anaya-AguirRe ${ }^{3}$ \\ Y ABRIL ADRIANA ARELLANO-LIAMAS ${ }^{4 *}$
}

${ }^{1}$ Servicio de Neonatología, Hospital de Ginecoobstetricia N. 3 , Instituto Mexicano del Seguro Social, Ciudad de México; ${ }^{2}$ Servicio de Pediatría, Hospital General de Zona 36 Coatzacoalcos, Instituto Mexicano del Seguro Social, Veracruz; ${ }^{3}$ Servicio de Oncología Pediátrica; ${ }^{4}$ Servicio de Endocrinología Pediátrica, Unidad Médica de Alta Especialidad, Hospital General La Raza, Instituto Mexicano del Seguro Social, Ciudad de México. México

\section{RESUMEN}

Los supervivientes de linfoma en edad pediátrica tienen desenlaces metabólicos adversos en la adultez. Conocer cómo se modifica el estado de nutrición medido por el índice de masa corporal (IMC) tras haber recibido manejo oncológico es un marcador indirecto del riesgo cardiovascular. Se estudió retrospectivamente a 30 pacientes pediátricos supervivientes de linfoma y se observó un incremento sostenido del valor del IMC estandarizado (szIMC) entre el inicio del tratamiento respecto al medido a seis meses de iniciar la vigilancia (szIMC: 0.11 vs. 1.04 desviaciones estándar [DE]; $\mathrm{p}<0.05$ ), más marcado en las mujeres (szIMC: -0.54 vs. $0.58 \mathrm{DE}$; $p<0.05)$. Se desarrolló hipotiroidismo en el $16 \%$ de los pacientes que recibieron radioterapia en el cuello. El seguimiento prospectivo en búsqueda de marcadores de riesgo cardiovascular en esta población es necesario para desarrollar manejo preventivo.

Palabras clave: Obesidad. Superviviente de cáncer. Paciente pediátrico.

\begin{abstract}
Survivors of childhood lymphoma have adverse metabolic outcomes in adulthood. The increase of body mass index (BMI) during childhood is an indirect cardiovascular risk marker. A retrospective observational study was conducted with 30 short term pediatric lymphoma survivors, standardized BMI increased between pre-chemotherapy and sixmonth post-treatment (0.11 vs. 1.04 standard deviation [SD]; $p<0.05)$; it was more noticeable in women (-0.52 vs. 0.58 SD; $p<0.05)$. Hypothyroidism was observed in $16 \%$ of those who received radiotherapy; all of them were opportunely treated. Prospective observation of other cardiovascular risk markers should be observed in this population.
\end{abstract}

Key words: Obesity. Cancer survivor. Pediatric patient.

\section{Correspondencia:}

*Abril Adriana Arellano-Llamas

E-mail: abrilarellano@yahoo.com.mx
Fecha de recepción: 24-09-2019

Fecha de aceptación: 06-11-2019

DOI: 10.24875/RME.19001952
Disponible en internet: 28-11-2019 Rev Mex Endocrinol Metab Nutr. 2019;6:165-9

2462-4144/@ 2019 Sociedad Mexicana de Nutricion y Endocrinologia, AC. Publicado por Permanyer México. Este es un artículo Open Access bajo la licencia CC BY-NC-ND (http://creativecommons.org/licenses/by-nc-nd/4.0/). 


\section{INTRODUCCIÓN}

El valor z (sz) del índice de masa corporal (IMC) expresa el número de desviaciones estándar que un individuo se aleja de la media y es la medida del estado de nutrición aceptado en los niños. Los rangos de normalidad más utilizados son las tablas de los Centers of Disease Control y de la Organización Mundial de la Salud. Los niños que presentan cambios rápidos en el IMC por alguna razón presentan mayor riesgo cardiovascular ${ }^{1}$.

El cáncer infantil es un problema de salud pública en México y es la segunda causa de muerte entre los niños de 4 a 15 años. El linfoma es el cáncer sólido más común. Los linfomas se presentan en los niños en sus tipos Hodgkin y no Hodgkin en el 52 y el $48 \%$ respectivamente ${ }^{2}$. De los subtipos de linfoma, el linfoma Hodgkin es el más común en los niños, con el 52.5\% de los casos. El tratamiento incluye quimioterapia, radioterapia o una combinación de ambas. El tratamiento de quimioterapia, con o sin radioterapia, logra la curación en más del $90 \%$ de los pacientes con esta enfermedad.

Diversos estudios han aportado observaciones sobre el efecto de haber padecido cáncer y la exposición a tratamiento oncológico en el IMC. En una cohorte de supervivientes de cáncer infantil se observó que los supervivientes de leucemia son más obesos, con una odds ratio (OR) de 1.5 (intervalo de confianza [IC] 95\%: 1.2-1.8); mientras los supervivientes de linfoma de Hodgkin presentan más desnutrición (OR: 1.7; IC 95\%: 1.3-2.3 $)^{3}$. Otras series que incluyen supervivientes de linfoma no han observado efecto sobre el IMC ${ }^{4}$.

Los supervivientes de linfoma en la edad pediátrica pueden tener mayor riesgo de morbimortalidad por causas cardiovasculares, por lo que resulta relevante estudiar los cambios en el IMC que puede presentar esta población después de haber alcanzado la remisión de la enfermedad ${ }^{5}$.

\section{MATERIAL Y MÉTODOS}

El presente estudio fue retrospectivo, descriptivo y analítico. Se realizó en la Clínica de Vigilancia de Cáncer del Servicio de Oncología del Hospital General
Tabla 1. Características generales de la muestra

\begin{tabular}{lcc}
\hline & $\mathrm{n}$ & $\%$ \\
\hline Sexo masculino & 18 & 60 \\
\hline Linfoma Hodgkin & 15 & 50 \\
\hline Estadio & & \\
2 & 10 & 35.7 \\
3 & 10 & 35.7 \\
4 & 8 & 28.6 \\
\hline Comorbilidades & 4 & 14.8 \\
\hline Hipotiroidismo posradioterapia & 5 & 16.7 \\
\hline
\end{tabular}

del Centro Médico La Raza, previa autorización del Comité de Investigación y Ética (R-2019-3502-012). Se incluyó la información de supervivientes de linfoma de 1 a 16 años y se comparó el valor $z$ del IMC al diagnóstico con el valor $z$ al inicio de la vigilancia y después de la remisión en intervalos de 6 meses.

Se calcularon las diferencias de las medias de los valores $z$ de la talla y del IMC al diagnóstico, al momento de la remisión y cada 6 meses durante la vigilancia. Se consideró como significativo un valor de $\mathrm{p}<0.05$. La población se describió con medidas de tendencia central.

\section{RESULTADOS}

De 42 pacientes supervivientes de linfoma, se obtuvo información de 30 expedientes. Las características generales se describen en la tabla 1. La mitad de los pacientes tuvieron diagnóstico de linfoma de Hodgkin, el $60 \%$ correspondió al sexo masculino; la edad media al diagnóstico fue de 9.94 años (desviación estándar [DE]: 3.44). En 17 pacientes (56\%) que recibieron esteroides, la dosis media fue de $2,950 \mathrm{mg}$ de dosis de cortisol. En la historia clínica de ingreso al hospital, se reportó la pérdida de peso como síntoma del cáncer en el $62 \%$ de los casos, la pérdida medida en mediana que las madres recordaron fue de $3 \mathrm{~kg}$ (1.7 a $4.0 \mathrm{~kg})$.

Los principales esquemas de quimioterapia utilizados en primera línea fueron bleomicina, etopóstido, doxorubicina, ciclofosfamida, vincristina, procarbazina y prednisona (BEACOPP), doxorurrubicina, bleomicina, 
Tabla 2. Evolución del valor z del índice de masa corporal (szIMC)

\begin{tabular}{|c|c|c|c|c|c|c|}
\hline szIMC & $\mathrm{n}$ & Mediana & $\begin{array}{c}\text { Rango } \\
\text { intercuartílico }\end{array}$ & $\mathrm{p}$ & $\begin{array}{l}\text { Mediana del } \\
\text { D szIMC }\end{array}$ & $\begin{array}{l}\text { Rango } \\
\text { intercuartílico } \\
\text { de la D szIMC }\end{array}$ \\
\hline Inicio del tratamiento & 30 & 0.05 & -0.87 a 0.95 & & 0 & \\
\hline Inicio de vigilancia & 30 & 0.83 & -0.23 a 1.68 & 0.01 & 0.46 & -0.40 a 1.41 \\
\hline Vigilancia 1 & 20 & 1.33 & 0.37 a 1.72 & 0.02 & 0.29 & -0.15 a 1.55 \\
\hline Vigilancia 2 & 14 & 0.94 & 0.33 a 1.71 & 0.01 & 0.34 & 0.11 a 1.76 \\
\hline Vigilancia 3 & 10 & 0.66 & -0.44 a 1.45 & 0.10 & 0.25 & -0.38 a 1.89 \\
\hline
\end{tabular}

Tabla 3. Evolución del valor z del índice de masa corporal (szIMC) en hombres

\begin{tabular}{|c|c|c|c|c|c|c|}
\hline szIMC & $\mathrm{n}$ & Mediana & $\begin{array}{c}\text { Rango } \\
\text { intercuartílico }\end{array}$ & $\mathrm{p}$ & $\begin{array}{l}\text { Mediana del } \\
\text { D szIMC }\end{array}$ & $\begin{array}{l}\text { Rango } \\
\text { intercuartílico } \\
\text { de la D szIMC }\end{array}$ \\
\hline Inicio del tratamiento & 18 & 0.29 & -0.25 a 1.71 & & 0 & \\
\hline Inicio de vigilancia & 18 & 1.25 & 0.41 a 1.80 & 0.08 & 0.33 & -0.40 a 1.41 \\
\hline Vigilancia 1 & 13 & 1.47 & 0.64 a 1.87 & 0.17 & 0.08 & -0.34 a 1.47 \\
\hline Vigilancia 2 & 7 & 1.41 & 0.45 a 1.75 & 0.02 & 0.40 & 0.16 a 1.62 \\
\hline Vigilancia 3 & 6 & 0.87 & -0.73 a 1.62 & 0.24 & -0.61 & -0.47 a 1.89 \\
\hline
\end{tabular}

Tabla 4. Evolución del valor z del índice de masa corporal (szIMC) en mujeres

\begin{tabular}{|c|c|c|c|c|c|c|}
\hline szIMC & $\mathrm{n}$ & Mediana & $\begin{array}{c}\text { Rango } \\
\text { intercuartílico }\end{array}$ & $\mathrm{p}$ & $\begin{array}{c}\text { Mediana del } \\
\text { D szIMC }\end{array}$ & $\begin{array}{l}\text { Rango } \\
\text { intercuartílico } \\
\text { de la D szIMC }\end{array}$ \\
\hline Inicio del tratamiento & 12 & -0.47 & -1.05 a 0.16 & & 0 & \\
\hline Inicio de vigilancia & 12 & 0.28 & -0.68 a 1.53 & 0.11 & 0.56 & -0.35 a 2.07 \\
\hline Vigilancia 1 & 7 & 0.41 & 0.18 a 1.53 & 0.09 & 1.25 & 0.06 а 2.18 \\
\hline Vigilancia 2 & 7 & 0.62 & -0.02 a 1.55 & 0.17 & 0.29 & -0.29 a 2.20 \\
\hline Vigilancia 3 & 4 & 0.44 & -0.35 a 1.17 & 0.35 & 0.25 & 0.25 a 2.78 \\
\hline
\end{tabular}

vinblastina y dacarbazina (ABVD), prednisona, ciclofosfamida, metotrexato, mercaptopurina(BFM), ciclofosfamida, doxorubicina, vincristina y prednisolona (CHOP), ciclofosfamida, vincristina, prednisona, metotrexate, doxorubicina, Ara C, etopósido (LMB), prednisona, vincristina, asparaginasa, metotrexate, mercaptopurina, araC, dexametasona (TOTAL), ifosfamida, carboplatino, etopósido (ICE). El 60\% de los pacientes presentaron eventos adversos, el más común fue fiebre y neutropenia (20\%). Solo un paciente requirió manejo en terapia intensiva durante el tratamiento del linfoma. De los ocho pacientes que recibieron radioterapia en el cuello, cinco (16\%) presentaron hipotiroidismo como complicación.
El szIMC incrementó de una mediana de $0.05 \mathrm{DE}$ al diagnóstico (rango intercuartílico: -0.87 a $0.95 \mathrm{DE}$ ) hasta una mediana 1.33 DE (rango intercuartílico: 0.37 a 1.72) al sexto mes de seguimiento; entre los hombres, de una mediana de 0.29 DE (rango intercuartílico: -0.25 a $1.71 \mathrm{DE}$ ) a $1.47 \mathrm{DE}$ (rango intercuartílico: 0.64 a $1.87 \mathrm{DE}$ ) y en mujeres de $-0.47 \mathrm{DE}$ (rango intercuartílico: -1.05 a $0.16 \mathrm{DE}$ ) a $0.41 \mathrm{DE}$ (rango intercuartílico: 0.18 a $1.53 \mathrm{DE}$ ) en el mismo periodo. No se observó modificación en los percentiles de la talla durante la vigilancia (Tablas 2-4 y Fig. 1).

Por la trascendencia clínica que significa incrementar una desviación estándar del IMC en la población pediátrica, clasificamos a la población de acuerdo 
Tabla 5. Características de la población que incrementó más de una desviación estándar (DE) tras seis meses de vigilancia

\begin{tabular}{|c|c|c|c|}
\hline Variable & $\begin{array}{l}\text { Pacientes que incrementaron } \\
\text { más de una } \mathrm{DE}(\mathrm{n}=8)\end{array}$ & $\begin{array}{l}\text { Pacientes que no incrementaron } \\
\text { más de una DE }(\mathrm{n}=13)\end{array}$ & $\mathrm{p}$ \\
\hline Dosis de cortisol acumulada & $25,880(20,160$ a 33,600$)$ & $8,100(7,200$ a 26,880$)$ & 0.99 \\
\hline Número de ciclos de quimioterapia & $6(4$ a 8$)$ & $8(6$ a 8$)$ & 0.367 \\
\hline Edad al diagnóstico & $7.7(5.45$ a 10.81$)$ & $13(9.41$ a 13.66$)$ & 0.183 \\
\hline Dosis de radioterapia & $32.5(25.5$ a 36$)$ & $30.6(30.6$ a 30.6$)$ & \\
\hline Hipotiroidismo primario & 3 & 0 & 0.142 \\
\hline Estadio de la enfermedad (III y IV) & 9 & 5 & 0.037 \\
\hline Sexo & & & 0.204 \\
\hline Masculino & 4 & 10 & \\
\hline Femenino & 4 & 3 & \\
\hline
\end{tabular}

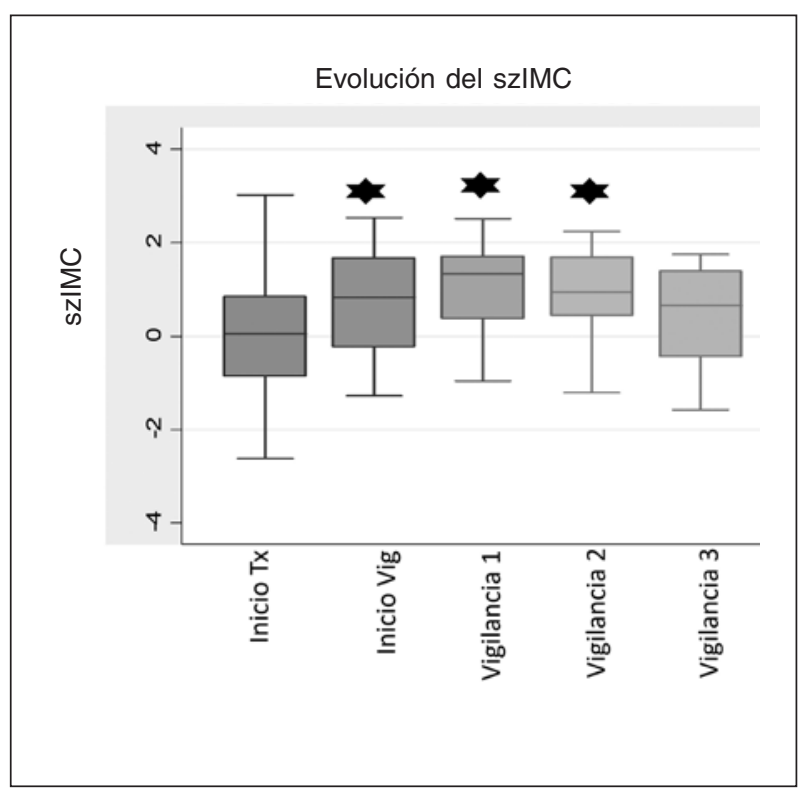

Figura 1. Gráfica de cajas sobre la evolución del valor z del índice de masa corporal (szIMC) desde el diagnóstico, al inicio de la vigilancia y cada seis meses posteriormente. Se observa un incremento significativo del szIMC al inicio de la vigilancia y en los primeros seis meses. Tx: tratamiento; Vig: vigilancia.

con este incremento a seis meses de vigilancia y observamos que no hubo diferencias en la dosis de esteroide recibido, padecer hipotiroidismo, el número de ciclos de quimioterapia recibidos, ni el sexo. En cuanto a la edad, si bien no hubo una diferencia estadísticamente significativa, es notorio que los pacientes más jóvenes (7.7 vs. 13 años) tendieron a incrementar más su IMC que los mayores $(p=0.18)$ (Tabla 5).

\section{DISCUSIÓN}

El aumento del IMC en supervivientes de cáncer se ha visto en otras poblaciones, considerándose que el tratamiento del cáncer puede tener efecto sobre el cerebro en desarrollo, alterar las vías de saciedad, provocar sobrealimentación y la consecuente obesi$\mathrm{dad}^{3}$. En este sentido se sabe que los supervivientes de leucemia tienen cuatro veces más riesgo de eventos cardiovasculares adversos, y significativamente mayor riesgo de desarrollar síndrome metabólico 2,3.

Las causas del incremento del szIMC pueden ser multifactoriales, y existe posibilidad de que el uso de quimioterapéuticos como los esteroides generen cambios epigenéticos que predispongan a cambios permanentes de la composición corporal ${ }^{4}$. El seguimiento a largo plazo de este grupo de pacientes dará luz a la persistencia o no de la ganancia de peso, y de la composición corporal como una línea de investigación prioritaria ${ }^{5}$. En este sentido, las historias clínicas que revisamos no contaban de forma sistemática con información relacionada con el ambiente nutricional de los pacientes como la existencia de obesidad en los padres, por ejemplo, ni los hábitos de alimentación o ejercicio antes, durante o después del tratamiento. En las notas de supervivencia no se registra el estado funcional de los pacientes ni su capacidad para realizar ejercicio, por lo que estos puntos son elementos indispensables de averiguarse prospectivamente para plantear un seguimiento integral de esta población, y representan 
para este estudio una debilidad para poder abordar otros aspectos relacionados con el desarrollo de la obesidad.

A pesar de que en la serie el $16 \%$ de los pacientes desarrollaron hipotiroidismo, todos tuvieron reemplazo hormonal oportuno y no se explica la modificación del IMC. En este sentido se sabe que el uso de radioterapia, de una manera dependiente de la dosis afecta la función tiroidea, habiendo efecto sobre esta hasta 25 años después de recibida la dosis en una proporción alta para los supervivientes de linfoma de Hodgkin, de hasta el 32\% ${ }^{6}$.

En esta serie no hubo afectación sobre el canal de crecimiento longitudinal de los pacientes.

Por ser este un estudio retrospectivo, existe posibilidad de que existan sesgos en la medición de la somatometría. En este sentido, es urgente realizar un seguimiento de cohorte sobre los supervivientes de cáncer infantil en nuestro país, lo que permitiría además añadir medición de la composición corporal y otros marcadores indirectos de riesgo cardiovascular que permitan ponderar los desenlaces posibles, así como también registrar variables asociadas a la obesidad como los antecedentes familiares, los hábitos de alimentación y la capacidad funcional previa y posterior al tratamiento.

Este estudio genera la necesidad de estudiar los efectos a mediano y largo plazo de los tratamientos quimioterapéuticos en cáncer sólido, así como el estudio de los desenlaces cardiovasculares en los supervivientes a largo plazo. Además, invita a conocer los efectos del cáncer y/o la quimioterapia sobre el funcionamiento metabólico y de saciedad de los pacientes.
El incremento progresivo del IMC entre los supervivientes de linfoma alerta sobre la necesidad de generar clínicas de atención para este grupo de pacientes que otorguen una atención multidisciplinaria que no se centre solo en la vigilancia de la supervivencia libre de enfermedad, sino que también considere los efectos secundarios del tratamiento subrayando los relacionados con el riesgo cardiovascular y el endocrinológico.

Concluimos que la observación del incremento del szIMC asociado a la supervivencia de linfoma requiere que se profundice en el estudio de las comorbilidades desencadenadas por la quimioterapia, y la generación de estrategias para influir en los desenlaces adversos que se presume ocurrirán.

\section{BIBLIOGRAFÍA}

1. Imai CM, Gunnarsdottir I, Gudnason V, Aspelund T, Birgisdottir BE, Thorsdottir $\mathrm{I}$, et al. Faster increase in body mass index between ages 8 and 13 is associated with risk factors for cardiovascular morbidity and mortality. Nutr Metab Cardiovasc Dis NMCD. 2014;24(7):730-6.

2. Mertens AC, Liu Q, Neglia JP, Wasilewski K, Leisenring W, Armstrong GT, et al. Cause-specific late mortality among 5-year survivors of childhood cancer: the Childhood Cancer Survivor Study. J Natl Cancer Inst. 2008;100(19):1368-79.

3. Miranda-Lora AL, Vilchis-Gil J, Molina-Díaz M, Flores-Huerta S, KlünderKlünder M. Heritability, parental transmission and environment correlation of pediatric-onset type 2 diabetes mellitus and metabolic syndrome-related traits. Diabetes Res Clin Pract. 2017:126:151-9.

4. Cutfield WS, Hofman PL, Mitchell M, Morison IM. Could epigenetics play a role in the developmental origins of health and disease? Pediatr Res. 2007;61 (5 Pt 2):68R-75R.

5. Touyz LM, Cohen J, Neville KA, Wakefield CE, Garnett SP, Mallitt KA, et al. Changes in body mass index in long-term survivors of childhood acute lymphoblastic leukemia treated without cranial radiation and with reduced glucocorticoid therapy. Pediatr Blood Cancer. 2017:64(4).

6. Inskip PD, Veiga LHS, Brenner AV, Sigurdson AJ, Ostroumova E, Chow EJ, et al. Hypothyroidism after radiation therapy for childhood cancer: $\mathrm{A}$ report from the Childhood Cancer Survivor Study. Radiat Res. 2018:190(2):117-32. 